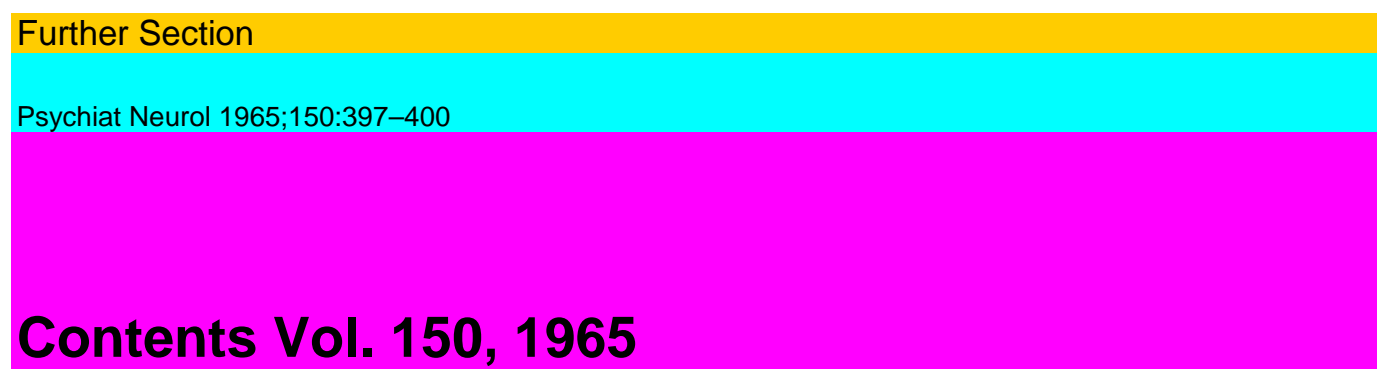

\title{
Psychiatria et Neurologia
}

Internationale Monatsschrift für Psychiatrie und Neurologie Revue Internationale Mensuelle de Psychiatrie et de Neurologie International Monthly Review of Psychiatry and Neurology

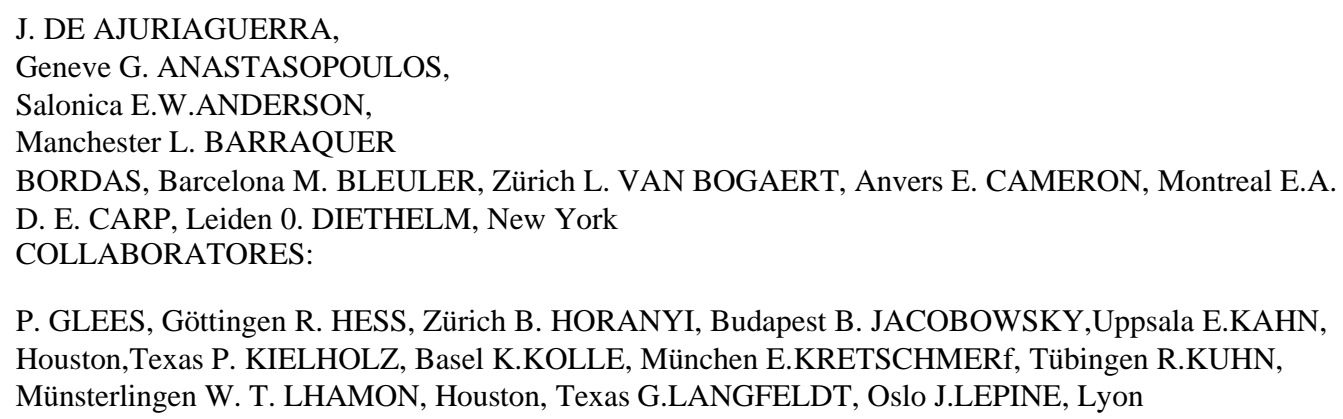

P. GLEES, Göttingen R. HESS, Zürich B. HORANYI, Budapest B. JACOBOWSKY,Uppsala E.KAHN, Houston,Texas P. KIELHOLZ, Basel K.KOLLE, München E.KRETSCHMERf, Tübingen R.KUHN, Münsterlingen W. T. LHAMON, Houston, Texas G.LANGFELDT, Oslo J.LEPINE, Lyon

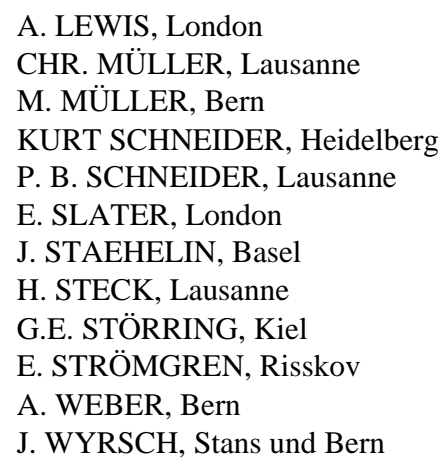

EDITOR: J. KLAESI, Schloß Knonau REDACTOR : E. GRÜNTHAL, Bern REDACTORES-SECRETARII: N. PETRILOWITSCH, Mainz TH. SPOERRI, Bern 


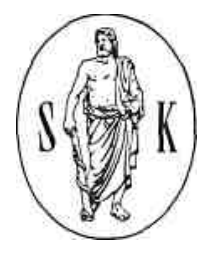

Vol. 150

1965

BASEL (Schweiz)
S. KARGER INDEX
NEWYORK

ABE, K.:

Patterns of Relapse in Remitting Psychotics

AJURIAGUERRA, J. DE; KLUSER, J.P.; Velghe, J. et Tissot, R.:

Praxies ideatoires et permanence de l'objet. Quelques aspects de leur desintegration conjointe dans les Syndromes dementieis du grand äge 306

- : vide CONSTANTINIDIS, J.

ALBERT, ELFRIEDE: Psychiatrische Beobachtungen an neuaufgenommenen Alterskranken

eines Landeskrankenhauses.

BRAND, NAPHTALI: Psychoneurosis in Four Collective Agricultural Settlements in Israel

CAMERON, D.E.: $\quad$ Uncovering in Psychotherapy

CANAL, N.: vide MASINI, T.

CENDROWSKI, W.S.: Rare Pedigrees in Multiple Sclerosis

CONSTANTINIDIS, J.; GARRONE, G.; TISSOT, R. et AJURIAGUERRA, J. DE:

L'incidence familiale des alterations neurofibrillaires corticales d'Alzheimer

FLAMENT-DURAND, J.: vide MARTIN, J. J.

GARRONE, G.: vide CONSTANTINIDIS, J.

GREGER, J.: vide WIECZOREK, V.

GuAZZI, G. C.: vide MASINI, T.

HENKING, R.: vide RALLO PIQUE, E.

IRÄNYI, C.: vide NYIRO, J.

KLTJSER, J. P.: vide AJURIAGUERRA, J. DE

LEWIS, A.:

A Note on Personality and Obsessional Hlness

MARTin, J. J. et FLAMENT-DURAND, J.:

Atrophies systematisees, etat marbre et dysplasie diffuse apparenteeä la sclerose tubereuse chez un meme sujet atteint de paraplegie spas-modique et d'oligophrenie.

MASINI, T.; CANAL, N. et GUAZZI, G. C.:

Poliomyelite chronique et degenerescence nigrique se presentant cli-niquement comme une amyotrophie progressive spinale 
MÜLLENER, E. R.: Zum Erscheinen von Band 150 der «Psychiatria et Neurologia». EinRückblick auf die Geschichte der «Monatsschrift für Psychiatrie undNeurologie»

NYIRO, J. and IRÄNYI, C:

A Contribution to the Interpretation of Ganser Symptoms

PILLERI, G.:

Über eine besondere normotone intracerebrale Gefäßerkrankung

(«Status cavernosus») mit Schwerpunkt im oberen Hirnstamm .. 358

RAIXO PIQUe, E. et HENKING, R.:

Experimentation clinique et electroencephalographicrue du diazepamintraveineux chez les malades epileptiques

SCHÄR, J.: $\quad$ Das Verhalten der Lipoprotein-Lipase bei multipler Sklerose und

anderen neurologischen Erkrankungen

SCHEHZER, E.: Über die gutachtliche Wertung des 4/sec-Rhythmus nach Schädeltraumen

SCHWAB, R. S. and ZIEPER, I.:

Effects of Mood, Motivation, Stress and Alertness on the Performance

in Parkinson's Disease

SCHWARTZ, PH. : Über Lokalisation und Ausdehnung zerebrospinaler Erkrankungen.Morphologische Eigenschaften, Pathogenese und allgemeine Bedeutung topistischer Reaktionen. 1

-------- : Über Lokalisation und Ausdehnung zerebrospinaler Erkrankungen.

Morphologische Eigenschaften, Pathogenese und allgemeine Bedeutung topistischer Reaktionen. II..

TISSOT, R.: vide AJTJRIAGUERRA, J. DE

: vide CONSTANTINEDIS, J.

VELGHE, J.: vide AJTJRIAGUERRA, J. DE

WALKER, A.E.: $\quad$ Frontal Lobe Epilepsy

WIECZOREK, V. und GBEGER, J.:

Erfahrungen mit der Licmorzelldiagnostik. Eine Analyse von etwa5000 liquorzytologischen Untersuchungen. I. Das normale Liquorzell-bild, Licruorzellbefunde bei primären und metastatischen Hirn- undRückenmarkgeschwülsten, bei zerebralen Gefäßerkrankungen sowienach Schädel-Hirntraumen

Erfahrungen mit der Licjuorzelldiagnostik. Eine Analyse von etwa

5000 liquorzytologischen Untersuchungen. II. Das Liquorzellbild beientzündlichen Hirn-Rückenmarkerkrankungen einschließlich dermultiplen Sklerose, bei neuroluischen Erkrankungen, nach frühkindlichen Hirnschäden, degenerativen Erkrankungen des ZNS, Epilepsieund endogenen Psychosen

ZIEPER, I.: vide SCHWAB, R. S.

LIBRI.

$56,118,184,248,320,384$

VARIA

INDEX HERUM ad Vol. 146-150

INDEX NOMINUM ad Vol. 146-150 
Alle Rechte, insbesondere das der Übersetzung in fremde Sprachen, vorbehalten.

Ohne ausdrückliche Genehmigung des Verlages ist es auch nicht gestattet, diesen Band oder Teile

daraus auf photomechanischem Wege (Photokopie, Mikrokopie) zu vervielfältigen.

Printed in Switzerland Glichet: Steiner \& Cie,, AG, Basel Druck der Basler Druck* und Verlags anstatt, BasC. Copyright 1965 by S. Karger AG

Basel 